\title{
ESTRATEGIAS EMPRESARIALES ENFOCADAS AL FORTALECIMIENTO DE LOS PROGRAMAS MOTIVADOS POR EL DESARROLLO ALTERNATIVO
}

\author{
Marly Julieth Rojas \\ Escuela de Negocios, Gestión y Sostenibilidad \\ Programa de Administración de Empresas \\ Administradora de Empresas \\ majurojasz@hotmail.es \\ Nathalia Parrado M \\ Escuela de Negocios, Gestión y Sostenibilidad \\ Programa de Administración de Empresas \\ Administradora de Empresas \\ nathalia.parrado90@gmail.com \\ Allynson Venegas Camargo \\ Escuela de Negocios, Gestión y Sostenibilidad \\ Programa Administración de Empresas \\ Docente \\ Magíster en docencia \\ vcallynson@poligran.edu.co
}

\section{Resumen}

Este trabajo toma como objeto de estudio la asociación Guardagolfo creada en el municipio de Necoclí y la asociación Bajo Mira y Frontera consejo comunitario del municipio de San Andrés de Tumaco, las cuales se desarrollaron en el marco del Desarrollo Alternativo como estrategia del gobierno para atacar los cultivos ilícitos. Se realiza una caracterización de las asociaciones partiendo de sus aspectos diferenciales en cuanto a los ámbitos económicos, políticos, sociales

y culturales de las regiones donde se encuentran, proponiendo estrategias empresariales que les permitan mejorar en su desempeño como organización. 


\section{Introducción}

Durante los últimos 50 años Colombia ha vivido una etapa de conflicto armado por la acción de diferentes grupos armados ilegales (Trejos Rosero, 2013, pág. 55), identificando a las guerrillas y los paramilitares como los más populares. Las consecuencias de la violencia han sido desastrosas, pues el $83 \%$ de la totalidad de los municipios del país se han visto afectados (CENTRO DE RECURSOS PARA EL ANÁLISIS DE CONFLICTOS, 2014).

Por otra parte, la propagación de cultivos ilícitos en los últimos años ha sido la fuente de financiación de estos grupos y una opción de ingresos económicos para muchas poblaciones en el país (Trejos Rosero, 2013, pág. 71).

Con el propósito de reducir y eliminar la presencia de cultivos ilícitos, el Gobierno Nacional con el apoyo de la Oficina de las Naciones Unidas contra la Droga y el Delito (UNODC) ha venido implementando como estrategia el Desarrollo Alternativo para combatir la expansión de los mismos mediante el convenio $\mathrm{COL} \mathrm{K} / 53$, promoviendo iniciativas productivas sostenibles y modelos organizativos para las comunidades más afectadas por la economía ilegal.

Este tipo de apoyo inició en entidades y programas del Gobierno Nacional como PLANTE PLAN COLOMBIA (DNP, 1995), Agencia Presidencial para la Acción Social y Cooperación Internacional (ACCIÓN SOCIAL) con su programa Presidencial contra Cultivos Ilícitos (PCI), el cual pasó a ser ejecutado por la Unidad Administrativa para la Consolidación Territorial (UACT) en el 2011 y hasta el 2015 y durante el 2016 la Dirección de Gestión Territorial de Departamento para la Prosperidad Social (PRESIDENCIA DE LA REPÚBLICA, 2015) lo ejecutó mediante el programa de Desarrollo Territorial y Sustitución de Cultivos Ilícitos. Bajo este programa se atendieron 2380 familias de los departamentos de Antioquia, Caldas, Cauca, Córdoba, Guainía, Guaviare, Magdalena, Putumayo y del archipiélago de San Andrés, providencia y Santa Catalina con una inversión de \$937'788.00 durante el año 2013 (ODC, 2017).

Basados en la información anterior, analizaremos el estado actual de dos organizaciones que surgieron en el marco del Desarrollo Alternativo, teniendo en cuenta los aspectos diferenciales en cuanto a la economía, ámbitos políticos, sociales y culturales, con el fin de proponer estrategias empresariales que les permitan mejorar sus procesos de producción, fortalecer su nivel organizacional y ampliar sus estrategias de comercialización.

\section{Contexto del desarrollo alternativo}

El Desarrollo Alternativo en Colombia ha permitido que diversas comunidades, familias y organizaciones por medio de la inclusión social y la integración territorial participen de manera activa en la creación y formación de empresas productoras de café, cacao, palma, caucho y diversos cultivos asociados. Así mismo, ha sido el medio para reducir y eliminar los cultivos ilícitos, mediante opciones de desarrollo rural diseñadas para cada una de las comunidades 
objetivo con base en sus características socioculturales especiales y con el objetivo de generar crecimiento a nivel nacional y un desarrollo sostenible para las comunidades (UNODC, 2017).

El monitoreo continuo realizado por la Oficina de las Naciones Unidas Contra la Droga y el Delito ha permitido identificar grandes beneficios en la aplicación del Desarrollo Alternativo como estrategia para generar oportunidades reales de desarrollo en las comunidades que han sido afectadas por las economías ilegales y que han permitido atender a más de 156.000 familias con iniciativas sostenibles en el largo plazo (UACT, 2013, pág. 9).

En efecto se evidencia la apuesta del Gobierno Nacional a la población campesina y demás vulnerada, con miras a reconocer su potencial y tradiciones agrícolas.

El Desarrollo Alternativo permite que las organizaciones reciban apoyo técnico durante el proceso de producción, a raíz de lo cual se ha detectado deficiencia en procedimientos como la recepción, el almacenamiento, la elaboración, el envase, empaque y transporte, considerándose oportunidades de mejora, como la posibilidad de transformar y generar un valor agregado en los productos que permita avanzar a los mercados finales y obtener mayores ganancias en el ejercicio (UNODC, 2017).

En consecuencia, el Desarrollo Alternativo como estrategia tiene como propósito promover la transición económica y social de la población objeto mediante propuesta licitas de desarrollo sostenible, con la finalidad de evitar la resiembra, persistencia y expansión de los cultivos ilícitos (UACT, 2015, págs. 3-4)

\section{Familias guardabosques: una estrategia de desarrollo alternativo}

El Programa Familias Guardabosques es una estrategia novedosa para el desarrollo alternativo de familias especialmente vinculadas con actividades relacionadas con cultivos ilícitos o que estuvieron en riesgo de involucrarse en ellos. Se han caracterizado por ser un tipo de familias nucleares o familias extendidas o extensas, las cuales se encuentran conformadas por 5 a 6 integrantes en promedio, donde la presencia de niños y adolescentes es significante (ACCIÓN SOCIAL/UNODC, 2007).

En cuanto a los niveles de escolaridad, apenas un 5\% para el año 2007 había completado la educación secundaria y tan solo el $1 \%$ ha hecho cursos de educación superior, lo que indica que presentan un alto nivel de analfabetismo (ACCIÓN SOCIAL/UNODC, 2007).

El tipo de tendencia de tierra para las familias guardabosques es un indicador puntualmente importante ya que se evidencia que un alto porcentaje de familias son poseedores y propietarios de sus tierras, aspecto que facilita en gran medida la inversión en sus propias tierras, pues sencillamente ningún guardabosque invertiría sus ahorros en un proyecto productivo que se desarrolla en tierras arrendadas, mucho menos si se trata de cultivos de larga duración como el caucho, el café, el cacao, los proyectos forestales, la palma o los proyectos de ecoturismo (ACCIÓN SOCIAL/UNODC, 2007).

Es necesario destacar que en la idea de avanzar hacia un país con más propietarios, un tema relevante se refiere a la compra masiva de tierras que ha propiciado el Programa. Entre los años 2004-2007, se observa que 11.405 Familias Guardabosques han logrado adquirir 43.378 hectáreas de tierra. Para este mismo período de tiempo el Instituto Colombiano de Desarrollo 
Rural (INCODER) adquirió 92.000 hectáreas de tierra en Colombia (ACCIÓN SOCIAL/UNODC, 2007).

En cuanto a los tipos de práctica que se vienen ejecutando, se puede identificar que el $15 \%$ se ha centrado en la rotación de cultivos, el $47 \%$ en la incorporación de rastrojos, el $37.5 \%$ en cero labranza, $8 \%$ en tala y quema y un último $12.5 \%$ en la siembra a través de la pendiente, prácticas realmente productivas, efectivas y ahorrativas (ACCIÓN SOCIAL/UNODC, 2007).

Un claro ejemplo de esto ha sido la implementación de prácticas amigables con el medio ambiente, las cuales han favorecido la estabilidad de los proyectos productivos. El 92\% de los beneficiarios afirman haber abolido dentro de sus actividades la tala y la quema en el establecimiento de sus cultivos. Para temas de saneamiento, el $60 \%$ arroja las aguas residuales directamente al suelo y cerca del $50 \%$ de los beneficiarios queman los papeles, cartones y plásticos (ACCIÓN SOCIAL/UNODC, 2007).

Otro tema corresponde al ahorro que las familias han logrado generar con el incentivo condicionado. Más de 34 mil millones de pesos ahorrados por familias campesinas y colonas de origen afrodescendiente, indígena, mestizo y blanco. Obtuvieron ingresos de $86,5 \%$ del incentivo condicionado, $69 \%$ de la producción de las fincas, el $32 \%$ del jornaleo agropecuario y el $99.9 \%$ de las familias guardabosques afirman que no retornarán a las actividades relacionadas con los cultivos ilícitos (ACCIÓN SOCIAL/UNODC, 2007).

Es así como el Programa Familias Guardabosques ha eliminado de manera manual y voluntaria gran cantidad de hectáreas de coca y de amapola otorgándoles a las sociedades llevar un estilo de vida más tranquilo y productivo.

Con el pasar de los años son más las familias inscritas dentro del programa de Familia Guardabosques en los diferentes departamentos todo con el fin de lograr que dichas familias gocen libremente de sus derechos fundamentales y constitucionales por medio del fortalecimiento de la participación ciudadana en la búsqueda del bienestar social en las regiones (UACT, 2017).

Las familias nucleares que lo componen se logra evidenciar que un tipo de familia nuclear se encuentra confirmada en promedio por 5 integrantes, mientras que las familias extendidas o extensas se componen de 6 integrantes en promedio (UNODC, 2010).

Las familias de los guardabosques están compuestas en su mayoría por niños y niñas y adolescentes (en el rango de edad entre 0 a 17 años) siendo esta participación del 46,5\%, seguido por un 38,7\% por los/las jóvenes y adultos jóvenes que se encuentran entre los 18 a 48 años. En último lugar se encuentra la población mayor de 49 años que ocupa el 14,8\% (UNODC, 2010).

\section{Modelo de graduación: premiando la asociatividad}

El modelo de graduación es una estrategia del Desarrollo Alternativo que atiende a aquellas familias localizadas en municipios de focalización, donde hay vulnerabilidad o presencia de cultivos ilícitos y que su población se ha visto afectada o es víctima de la violencia y conflicto 
armado en Colombia. Estas familias se encuentran asociadas, unidas o agrupadas en torno a una actividad productiva desarrollada en el marco del Desarrollo Alternativo, a través de acciones que pueden ser articuladas con otras entidades del Estado, de Cooperación Internacional y/o del sector privado, con el fin de promover el encadenamiento productivo, la capacidad de autogestión y su consolidación en el marco de la legalidad (UACT, 2015, pág. 4).

Entre los objetivos del modelo de graduación se evidencia la importancia de fortalecer las organizaciones en los aspectos de producción, además como una forma de generar desarrollo en las regiones y permitir la participación de las comunidades en la economía (UACT, 2015, pág. 5).

Es un requisito primordial que quienes sean beneficiados por este modelo sean Asociaciones, Comités departamentales y/o municipales, Agremiaciones, Cooperativas, Organizaciones de segundo nivel, Juntas de Acción Comunal-JAC, Comunidades Indígenas, Comunidades Negras, Afrocolombianas, Raizales y Palenqueras debidamente constituidas por familias que han sido apoyadas por el programa de Familias Guardabosques, para lo cual deberán presentar una iniciativa productiva que será sometida a evaluación y elección (UACT, 2015).

Otro requisito y obligación de gran importancia radica en la dejación total de los cultivos ilícitos, por cuanto los territorios de las familias deben estar libres de los mismos. Solo para el caso de los resguardos indígenas y en consideración con sus prácticas ancestrales se hace necesaria una consulta previa, siendo esta un dialogo que busca garantizar la participación real, oportuna y efectiva de los grupos étnicos en la toma de decisiones de proyectos que los afecten de tal forma que se proteja su identidad étnica y cultural (MINISTERIO DEL INETRIOR, 2013), la misma debe ser adelantada por el Ministerio de Interior (UACT, 2015).

\section{Caracterización de la asociación guardagolfo}

Con base en lo anterior, encontramos dentro del territorio colombiano, en los lugares mayormente afectados por la violencia, conflicto armado y presencia de cultivos ilícitos, una serie de organizaciones de la economía solidaria que desarrollan actividades, en su mayoría dentro del sector agrario, con fines de desarrollo económico y social. Estas organizaciones deben desarrollar habilidades y competencias para sobrevivir dentro del mercado, más aún con la realidad económica del país.

Dentro de la investigación realizada, evidenciamos que la mayoría de las debilidades de las organizaciones están relacionadas con la planeación estratégica, el cumplimiento de los aspectos legales, el control de calidad, los procesos logísticos y la falta de innovación, entre otros. Por otra parte, encontramos que con la implementación de los acuerdos de paz con la guerrilla de las FARC y en línea con el Plan de Desarrollo Nacional 2014 - 2018, el Gobierno realizará inversiones en los municipios donde se encuentran establecidas las organizaciones y que tienen como objetivo desarrollar la economía del país en el sector agrario. Así las cosas, en términos de oportunidades, las organizaciones que nacieron en el marco del Desarrollo Alternativo pueden encontrar apoyo en los diferentes proyectos a implementar, de forma tal que disminuyan en gran proporción las debilidades encontradas.

Otra gran debilidad está relacionada con la tecnificación de los procesos y el uso de las TIC's en ello. Estas organizaciones tienen grandes ventajas en cuanto al producto que desarrollan, 
ya que cada producto es autóctono de su zona y se cultiva en las mejores condiciones. Es importante lograr que el proceso de producción sea eficiente, logrando optimizar al máximo los recursos en tiempo y costos, es decir, estas organizaciones tienen el reto de incluir nuevas tecnologías para lograr mejorar sus procesos, ofreciendo la misma calidad o aún mejor, generando ventajas competitivas, incluso con miras a incursionar en mercados internacionales.

El estado actual de estas organizaciones no es el mejor, puesto que, al pertenecer al sector de la economía solidaria, no buscan un lucro, sino que, a través del desarrollo de una actividad economía se vean beneficiados los asociados en cuanto a si bienestar y economía. Por tanto, encontramos un bajo compromiso de los asociados, quienes al no ver grandes beneficios disminuyen o cortan sus aportes, dificultando el desarrollo y crecimiento de la organización.

A pesar de que las organizaciones pertenezcan al sector de la economía solidaria, no quiere decir que no deben generar ganancias, todo lo contrario, entre más ganancias generen mayor será el desarrollo económico de la población, siendo este uno de los objetivos principales del Desarrollo Alternativo. Entonces, estas organizaciones tienen la obligación de verse y desarrollar el mismo diagnóstico y análisis empresarial que desarrollaría cualquier empresa del sector real.

\section{Conclusiones.}

Como resultado de la investigación presentada, es posible concluir que en relación a cada cultura, costumbres y tendencias se evidencian diferencias dentro de las organizaciones en cuanto a la forma en que realizan la gestión y llevan a cabo su actividad económica.

Igualmente, se evidencia en las dos organizaciones que la falta de conocimiento técnico sobre conceptos básicos para la creación y el desarrollo de empresa han sido una de las problemáticas y mayores obstáculos que imposibilitan el buen desempeño y el alcance puntual de los objetivos.

El índice de analfabetismo derivado del mal manejo de los recursos por parte de los gobernantes, la mínima cantidad de recursos destinados a la sostenibilidad de la economía de las personas y de las empresas, la falta de apoyo por parte del gobierno dentro del desarrollo, la pésima infraestructura de las vías, el bajo conocimiento sobre procedimientos, normas, leyes en aspectos ambientales, legales y gubernamentales, son otras de las causas evidenciadas que impiden un excelente rendimiento y el alcance de objetivos.

El desarrollo alternativo ha sido una herramienta para la erradicación cultivos ilícitos en la región de Tumaco y Urabá en los departamentos de Nariño y Antioquia respectivamente, que durante su intervención permitió la conformación de diferentes tipos de organizaciones del sector de la economía solidaria como las analizadas durante esta investigación, donde se incentiva el desarrollo económico y social con la puesta en marcha de un proyecto de negocio y mediante el aprovechamiento de los recursos naturales de cada región, bajo la cultura de la legalidad. Sin embargo, se evidencia una falencia en esta estrategia, ya que su enfoque no considera que las organizaciones tienen presentan grandes debilidades a nivel interno, como la falta de sistemas de gestión, control de procesos, control contable, desarrollo del talento humano, entre otros, que hacen que su desarrollo sea más lento y poco probable. 
La misión y visión planteada por las asociaciones es clara, coherente y de gran enfoque, no obstante, es necesario un análisis de la organización acompañado de nuevas estrategias que se ajusten a las problemáticas de la organización y que permitan el desarrollo gradual y constante de la idea de negocio, así mismo su competitividad dentro del mercado.

Las estrategias obtenidas del desarrollo de la matriz DOFA para cada una de las organizaciones son posibles recomendaciones que desde nuestra perspectiva podrían ser un inicio para el fortalecimiento y crecimiento organizacional.

Evidentemente estas asociaciones son excelentes ideas de negocio, sobre todo porque se basan en el aprovechamiento de los recursos de cada región, por cuanto tienen un gran potencial de explotación.

En cuanto a las estrategias planteadas para la Asociación Guardagolfo se propone beneficiarse de los programas y proyectos que ofrece la Alcaldía de Necoclí, buscando un apoyo económico que les permita mejorar su capital de trabajo, siendo el mayor impedimento para poder ampliar su capacidad de gestión. Por otra parte, la asociación debe implementar la innovación tanto dentro de sus procesos como en los productos que comercializa y los servicios que ofrece.

Por otro lado, y enfocados en la asociación Bajo Mira y frontera se plantearon estrategias enfocadas a la apertura de nuevos mercados, haciendo provecho de las fortalezas y las certificaciones obtenidas. Generar un valor agregado en los productos y un reconocimiento de marca otorgara continuidad en los controles de calidad de sus suelos y productos con el fin de obtener excelencia en procesos y procedimientos. Incursionar en tecnología e innovación para los controles financieros generara un mayor desempeño y control financiero. Es una asociación que se caracteriza por comercializar producto de excelente calidad permitiendo ser parte de una de las redes cacaoteras más grandes del país, lo que le permitirá expandirse a gran escala dentro del mercado. 


\section{Bibliografía}

(s.f.).

ACCIÓN SOCIAL/UNODC. (2007). INFORME EJECTIVO SOBRE EL SEGUIMIENTO A LOS PROGRAMAS DE FAMILIAS GUARDABOSQUES Y PROYECTOS PRODUCTIVOS. BOGOTA.

ACNUR. (AGOSTO de 2004). ALGUNOS INDICADORES SOBRE LA SITUACIÓN DE LOS DERECHOS HUMANOS EN LA REGIÓN DEL URABÁ ANTIOQUEÑO. Obtenido de AGENCIA DE LA ONU PARA LOS REFUGIADOS: http://www.acnur.org/t3/uploads/media/COI_675.pdf

ALCALDÍA DE NECOCLÍ. (2017). ALCALDÍA DE NECOCLÍ. Obtenido de http://www.necocliantioquia.gov.co/MiMunicipio/Paginas/Economia.aspx

ALCALDÍA DE TUMACO. (2016). Plan de Desarrollo Municipal 2016 - 2019 "Tumaco Nuestra Pasión". "Tumaco Nuestra Pasión". Tumaco, Colombia.

ALCALDIA DE TUMACO. (2 de MAYO de 2017). ALCALDIA DE TUMACO. Obtenido de http://www.tumaco-narino.gov.co/informacion_general.shtml

ALCALDIA MUNICIPAL NECOCLÍ. (2016). PLAN MUNICIPAL DE DESARROLLO "POR UN BUEN GOBIERNO". PLAN MUNICIPAL DE DESARROLLO "POR UN BUEN GOBIERNO". COLOMBIA.

Andrews, K. (1977). El concepto de estrategia en la empresa. Pamplona: Univerisdad de Navarra.

Ansoff, H. (1976). La estrategia de la empresa. Pamplona: Universidad de Navarra.

CAMARA DE COMERCIO DE URABÁ. (2016). INFORME SOCIO ECONÓMICO 2015.

CENTRO DE RECURSOS PARA EL ANÁLISIS DE CONFLICTOS. (ABRIL de 2014). CERAC. Obtenido de http://www.cerac.org.co/es/1\%C3\%ADneas-de-investigaci\%C3\%B3n/analisisconflicto/tipologia-por-municipios-del-conflicto-armado.html

DANE. (2005). BOLETÍN - Censo General 2005 Perfil Necoclí - Antioquia.

Departamento Nacional de Planeación. (2012). Planeación para el desarrollo integral en las entidades territoriales. El plan de desarrollo 2012-2015. Bogotá, Colombia: Imprenta Nacional de Colombia.

DNP. (23 de Agosto de 1995). PLAN NACIONAL DE DESARROLLO ALTERNATIVO II PLANTE. CONPES 2799. Bogotá, Colombia.

DNP. (7 de Febrero de 1996). PLAN NACIONAL DE DESARROLLO ALTERNATIVO PLANTE. CONPES 2835. Bogotá, Colombia.

Forrester, I. (1972). Dinámica Industrial. Buenos Aires: El ateneo.

Fred R, D. (2013). Conceptos de Administración Estratégica. México: Pearson.

H.I., A. (1976). La estrategía de la empresa. La estrategía de la empresa . Pamplona : Universidad de Navarra.

Kast, F., \& Rosenzweight, J. (1978). Administración en la organizaciones. Enfoque de sistemas y de contigencia. México: Mc. Graw-Hill. 
Martinez Pedróz, D., \& Milla Gutierrez, A. (2012). Análisis del Entorno. Madrid: Ediciones Diaz de Santos.

MINISTERIO DEL INETRIOR. (2013). MININTERIOR. Obtenido de http://www.upme.gov.co/Memorias\%20Convocatoria\%20Redes\%20de\%20Alto\%20Voltaje/ MININTERIOR_CONSULTA-PREVIA.pdf

ODC. (Mayo de 2017). Observatorio de Drogas de Colombia. Obtenido de http://www.odc.gov.co/sidco/oferta/familias-guardabosques

Porter, M. (1985). Estrategia competitiva. Piramide.

PRESIDENCIA DE LA REPÚBLICA. (30 de Diciembre de 2015). DECRETO 2559. Bogotá, Colombia.

PRESIDENCIA DE LA REPÚBLICA. (29 de JULIO de 2016). PRESIDENCIA DE LA REPÚBLICA DE COLOMBIA. Obtenido de http://es.presidencia.gov.co/noticia/160729Asociacion-Guarda-Golfo-nominada-a-premio-internacional-de-emprendimiento-social

Red Cacaotera. (2 de MAYO de 2017). RED CACAOTERA . Obtenido de http://www.redcacaotera.com/index.php/es/asociados/presencia-en-colombia/nodosur/consejo-comunitario-bajo-mira-y-frontera-de-tumaco-narino

Red ORMET / PNUD. (2015). Perfil productivo Municipio de Necoclí (Antioquia). Programa de las Naciones Unidas Para el Desarrollo.

Robbins, S. (2016). Administración Décima edicion.

SINIC. (2 de MAYO de 2017). Sistema Nacional de Información Cultural. Obtenido de http://www.sinic.gov.co/SINIC/ColombiaCultural/ColCulturalBusca.aspx?AREID=3\&SECI $\mathrm{D}=8 \& \mathrm{IdDep}=52 \& \mathrm{COLTEM}=214$

Tabatoni, P., \& Jamiou, P. (1975). Les systémes de gestion. Politiques er structures . Paris: PUF.

Trejos Rosero, L. F. (2013). COLOMBIA: UNA REVISIÓN TEÓRICA DE SU CONFLICTO ARMADO. Revista Enfoques $\bullet$ Vol. XI $\bullet N^{o} 18 \bullet$, pp . 55-75.

UACT. (2007). INFOME ALIMENTEC. BOGOTA.

UACT. (2013). INFORME EJECUTIVO ENCUENTRO NACIONAL DE DESARROLLO ALTERNATIVO 2013. BOGOTA.

UACT. (2014). CONSOLIDANDO TERRITORIOS PARA LA PAZ. BOGOTA: IMPRENTA NACIONAL.

UACT. (27 de MARZO de 2014). UNIDAD ADMINISTRATIVA PARA LA CONSOLIDACION TERRITORIAL. Obtenido de Programa Contra Cultivos Ilícitos:

http://www.consolidacion.gov.co/themes/danland/descargas/entidad/PCI/00139_MODIFICA CION_RESOLUC_00366_2012.pdf

UACT. (11 de MARZO de 2015). MANUAL TÉCNICO OPERATIVO MODELO DE DE POSTERRADICACIÓN Y CONTENCIÓN - FAMILIAS GUARDABOSQUES PARA LA PROSPERIDAD . BOGOTA, COLOMBIA: UNIDAD ADMINISTRATIVA PARA LA CONSOLIDACIÓN TERRITORIAL. 
UACT. (2015). MANUAL TÉCNICO OPERATIVO MODELO DE GRADUACIÓN FORTALECIMIENTO PRODUCTIVO . MANUAL TÉCNICO OPERATIVO MODELO DE GRADUACIÓN - FORTALECIMIENTO PRODUCTIVO . BOGOTA.

UACT. (2016). Caracterización de organizaciones de Desarrollo Alternativo basado en su capacidad organizacional. Bogotá.

UACT. (2016). INFORME DE CARACTERIZACIÓN DE ORGANIZACIONES DE DESARROLLO ALTERNATIVO BASADO EN SU CAPACIDAD ORGANIZACIONAL . IMPRENTA NACIONAL.

UACT. (MAYO de 2017). UNIDAD ADMINISTRATIVA PARA LA CONSOLIDACIÓN

TERRITORIAL. Obtenido de http://www.consolidacion.gov.co

UNODC. (2010). DES-CIFRANDO LAS FAMILIAS GUARDABOSQUES . Bogotá: Impresol ediciones Ltda.

UNODC. (2017). Naciones Unidas contra la Droga y el Delito. Obtenido de https://www.unodc.org/colombia/es/news/guardagolfo.html

UNODC. (MAYO de 2017). Naciones Unidas Contra la Droga y el Delito. Obtenido de United Nation Office on Drugs and Crime:

https://www.unodc.org/colombia/es/da/fortalecimientocomercial.html 